\title{
Incremento en la proteína no degradable en rumen de vacas lecheras. 2. Efectos sobre utilización y excreción del nitrógeno ${ }^{\#}$
}

\author{
Increasing of rumen undegradable protein in dairy cows. \\ 2. Effects on nitrogen utilization and excretion
}

\author{
D Ibarra ${ }^{1 *}$, L Latrille ${ }^{1}$, F Wittwer ${ }^{2}$ \\ ${ }^{1}$ Instituto de Producción Animal, Facultad de Ciencias Agrarias, Universidad Austral de Chile, Casilla 567, Valdivia, Chile. \\ ${ }^{2}$ Instituto de Ciencias Clínicas, Facultad de Ciencias Veterinarias, Universidad Austral de Chile, Casilla 567, Valdivia, Chile.
}

SUMMARY

Eight confined lactating cows at mid lactation fed diets based on silages were used in an incomplete Latin square design to evaluate the effects of increasing rumen undegradable protein (RUP) on nitrogen utilization and excretion. Four diets were used. Diets designed as A1 and B1 had, on a DM basis, $45 \%$ pasture silage and 20\% alfalfa haylage, while diets A2 and B2 had 38\% permanent pasture and 27\% alfalfa silage. Diets A and B differed mainly on the level of RUP. Diets A were formulated accepting up to a 20\% RUP deficit and included soybean meal, while B diets covered $100 \%$ of estimated RUP requirements, using fish meal as a protein source. In spite of causing a lower $\mathrm{N}$ intake $(\mathrm{P}<0.05)$, diets higher in RUP $(\mathrm{B}$ diets) resulted in similar milk production. Productive $\mathrm{N}$ (secreted as milk nitrogen plus $\mathrm{N}$ in daily gain, expressed as $\mathrm{g} \mathrm{day}^{-1} \mathrm{and} \mathrm{as} \mathrm{g} \mathrm{kg}^{-1}$ of $\mathrm{N}$ intake) was improved significantly $(\mathrm{P}<0.05)$ in higher RUP diets. Faecal and urinary $\mathrm{N}$ excretion were also lower $(\mathrm{P}<0.05$ and $\mathrm{P}<0.01$, respectively) in these diets. Intake $\mathrm{N}$ used in productive functions was higher in $\mathrm{B}$ diets $(\mathrm{P}<0.01)$. The relationship productive $\mathrm{N}$ v/s intake $\mathrm{N}$ and excreted $\mathrm{N}$ (faeces plus urine) per litre of milk produced, and productive $\mathrm{N}$ v/s excreted $\mathrm{N}$ were all improved with $\mathrm{B}$ diets. These data indicate that correcting a RUP deficit of high silage diets with fish meal, increased the efficiency of the use of $\mathrm{N}$ for productive functions and decreased potential contamination through a reduced $\mathrm{N}$ excretion.

Palabras clave: ensilaje, utilización nitrógeno, excreción.

Key words: silage, nitrogen utilization, excretion.

\section{INTRODUCCION}

En los últimos años la producción de leche ha tenido un crecimiento importante no solo a nivel regional, sino también a nivel mundial. A pesar de las necesidades de aumentar la producción de alimentos, en los países desarrollados hay una preocupación creciente por que esta producción se desarrolle en forma sustentable (Spedding 1995). Esta necesidad se basa en que el deterioro del medio ambiente es ocasionado en parte por las excreciones generadas por este sistema productivo, ya sea por metano y $\mathrm{CO}_{2}$ que contribuyen al "efecto invernadero", como por las heces y orina, que al eliminar $\mathrm{N}$ y $\mathrm{P}$ pueden contaminar el suelo y, especialmente, las aguas subterráneas (Tamminga 1992; Tamminga 1996). Este problema ha cobrado tal magnitud que existen autores (Pell 1992) que plantean que las lecherías deberían limitar su tamaño a su capacidad de procesar sus purines.

Aceptado: 03.03.2006.

\# Financiación: Fondecyt (proyecto 1961045).

* Dirección actual: Departamento de Reproducción Animal, Facultad de Veterinaria, Universidad de la República, Lasplaces 1550, Montevideo, Uruguay. E-mail: dibarra@adinet.com.uy
Muchos países han legislado sobre el tema (Pell 1992; Tamminga 1996), estableciendo límites en el tamaño del rebaño basados en su potencial de contaminación. En este mismo sentido, también existen trabajos que intentan modelar las excreciones de N (Dou y col 1996, Van Straalen 1995) para optimizar el manejo de los purines.

La orina es, desde el punto de vista medioambiental, más crítica, ya que en ella el nitrógeno se encuentra principalmente como urea. En el medio ambiente existe una alta proporción de microorganismos con actividad ureásica, por lo que el $\mathrm{N}$ urinario es fácilmente volatilizado a la atmósfera como amoníaco. Los nitratos, por otra parte, generan contaminación de aguas subterráneas (Van Vuuren y col 1993, Tamminga 1996).

Por lo anterior, se realizó este trabajo con el objetivo de evaluar si al corregir un déficit de proteína no degradable en el rumen (PNDR) mediante el uso de harina de pescado, se mejora la utilización del N, en vacas lecheras confinadas alimentadas con dietas ricas en ensilajes.

\section{MATERIAL Y METODOS}

DISEÑO EXPERIMENTAL. El experimento se desarrolló en un predio experimental de la Universidad Austral de 
Chile, ubicado en Valdivia, Chile ( $\left.39^{\circ} 47^{\prime} \mathrm{LS} ; 7^{\circ} 13^{\prime} \mathrm{LO}\right)$. Constó de dos períodos de 14 días de duración que se efectuaron entre el 24 de enero y el 7 de febrero y entre 7 y el 21 de febrero de 1997. Los primeros 8 días de cada período se utilizaron para acostumbrar a los animales al cambio de dieta y los resultados utilizados se refieren a los 6 últimos días de cada uno de los dos períodos descritos. Ocho vacas Holstein-Friesian, con parto de invierno y de 17 a 22 semanas de lactación fueron agrupadas en dos cuaternas homogéneas en un diseño de dos cuadrados latinos incompletos (Gill 1981). Estos animales tenían una producción al inicio del ensayo de 26,0 \pm 3,5 L día ${ }^{-1}$ y su peso era de $542 \pm 36,9 \mathrm{~kg}$. Los animales se estabularon en un galpón con comederos y bebederos individuales y con libre acceso al agua y a la ración, suministrada para tener un $10 \%$ de rechazo. Los animales fueron alimentados 2 veces al día, en cantidades iguales y luego de cada ordeña, en un sistema de ración total mezclada. En el caso de que los animales consumieran la totalidad de la ración, al día siguiente esta era incrementada en un 5\%. Las temperaturas extremas, fueron de 7,3 y $19,7^{\circ} \mathrm{C}$ para el primer período y de 9,5 y $22,1^{\circ} \mathrm{C}$ para el segundo.

FORMULACIÓN Y EVALUACION DE LAS DIETAS. Se formularon 4 dietas isoenergéticas e isoproteicas, dos con bajo contenido de PNDR (A1 y A2) y dos con alto contenido de PNDR (B1 y B2) (cuadro 1). Las dietas tuvieron una relación ensilajes: concentrado de 65:35 base materia seca (BMS), empleando ensilajes de pradera $45 \%$ y alfalfa $20 \%$ en las dietas A 1 y B 1 о $38 \%$ y $27 \%$ respectivamente en las dietas A2 y B2. El mayor contenido de PNDR de las dietas B1 y B2 se obtuvo incorporando harina de pescado en sustitución del afrecho de soya de las dietas A1 y A2. Las dietas A1 y A2 fueron formuladas aceptando un $20 \%$ de déficit en la recomendación de PNDR, pero las dietas B1 y B2 aportaron el 100\% de este requerimiento (NRC 1989). Los valores de PNDR fueron obtenidos de una tabla de composición de alimentos generada localmente (Anrique y col 1995), considerando el contenido de PNDR de todos los alimentos incluidos en la dieta. Las diferencias entre ambas formas de formular se obtuvieron variando la composición de los concentrados

COLECCIONY ANALISIS DE MUESTRAS. La colección y análisis de muestras se realizó diariamente durante los últimos 5 días de cada período.

Alimentos. Tanto los concentrados como los ensilajes ofrecidos a cada animal, al igual que los rechazos, fueron pesados diariamente. Debido a que los animales utilizados eran parte de un ensayo de producción (Ibarra y Latrille 2006) se tomaron los resultados de composición de los alimentos de ese ensayo. En el laboratorio, se determinó MS del ensilaje a $105^{\circ} \mathrm{C}$ por secado en horno.
Cuadro 1. Composición de ingredientes y nutrientes de las dietas utilizadas (\% base MS).

Ingredients and nutrient composition of the diets (\% DM basis).

\begin{tabular}{|c|c|c|c|c|c|}
\hline Dieta & & A 1 & B 1 & A 2 & B 2 \\
\hline Nivel PNDR & & Bajo & Alto & Bajo & Alto \\
\hline \multicolumn{6}{|l|}{ Ingredientes } \\
\hline $\begin{array}{l}\text { Ensilaje de pra- } \\
\text { dera (PP) }\end{array}$ & $\%$ & 45 & 45 & 38 & 38 \\
\hline $\begin{array}{l}\text { Ensilaje de alfal- } \\
\text { fa (AA) }\end{array}$ & $\%$ & 20 & 20 & 27 & 27 \\
\hline Maíz & $\%$ & 15,0 & 15,0 & 15,3 & 15,7 \\
\hline Avena & $\%$ & 7,0 & 7,6 & 6,6 & 6,2 \\
\hline Triticale & $\%$ & - & - & - & 2,7 \\
\hline $\begin{array}{l}\text { Harina de } \\
\text { pescado }\end{array}$ & $\%$ & - & 4,7 & - & 4,7 \\
\hline Afrecho de soja & $\%$ & 5,3 & - & 5,1 & - \\
\hline Corn gluten meal & $\%$ & 7,8 & 7,0 & 7,2 & 4,9 \\
\hline Mezcla mineral & $\%$ & 0,8 & 0,8 & 0,9 & 0,8 \\
\hline \multicolumn{6}{|l|}{ Nutrientes } \\
\hline MS & $\%$ & 45,9 & 46,1 & 47,0 & 47,3 \\
\hline $\mathrm{CP}$ & $\begin{array}{l}\% \\
\text { MS }\end{array}$ & 15,3 & 15,6 & 15,8 & 16,2 \\
\hline PNDR & $\% \mathrm{PC}$ & 25,3 & 31,1 & 24,9 & 29,6 \\
\hline ENL & $\begin{array}{c}\text { Mcal/ } \\
\mathrm{kg}\end{array}$ & 1,6 & 1,6 & 1,6 & 1,6 \\
\hline FDA & $\begin{array}{c}\% \\
\text { MS }\end{array}$ & 28,9 & 22,1 & 28,9 & 28,2 \\
\hline FADIN & $\begin{array}{c}\% \\
\text { MS }\end{array}$ & 1,1 & 1,1 & 1,1 & 1,0 \\
\hline FND & $\begin{array}{l}\% \\
\text { MS }\end{array}$ & 44,1 & 45,1 & 43,5 & 43,1 \\
\hline FNDIN & $\begin{array}{c}\% \\
\mathrm{MS}\end{array}$ & 0,97 & 1,06 & 1,05 & 1,21 \\
\hline
\end{tabular}

Para la cuantificación de PC se utilizó la técnica de Kjeldhal de medición indirecta basada en una digestión ácida siendo el factor de conversión de $\mathrm{N}$ a proteína de 6.25 (AOAC 1996). La EM se estimó a partir de una regresión determinada localmente del valor D (materia orgánica digestible) establecida in vitro según Tilley y Terry (1963). La determinación de FDN se realizó de acuerdo a Van Soest y col (1991). Del rechazo de cada animal se tomaba una muestra diaria de aproximadamente $100 \mathrm{~g}$ y se hacía un pool semanal en el que se determinaba MS en horno a $105^{\circ} \mathrm{C}$ y $\mathrm{PC}$ según Kjeldahl (AOAC 1996) para restarla de la MS ofrecida y obtener el consumo de MS y N de cada vaca. Los valores de degradabilidad en rumen de la proteína corresponden a cifras tabuladas (Anrique y col 1995). 
Leche. Los volúmenes de leche diarios fueron medidos individualmente en cada ordeña (6 y 16:30 horas) tomándose muestras individuales de aproximadamente $50 \mathrm{cc}$ en la mañana y en la tarde para la determinación de grasa, proteína y urea. Estas muestras eran tomadas desde los medidores de leche, previa homogeinización del contenido mediante la entrada de aire. Las muestras fueron preservadas con dicromato de potasio y refrigeradas a $4^{\circ} \mathrm{C}$ hasta ser enviadas al laboratorio. Tanto la proteína verdadera como la grasa fueron determinadas con un aparato Milk-o-Scan sistema 4000, serie 300 (Fosselectric, Dinamarca). La urea fue determinada por el método ureasaBerthelot modificado (Wittwer y col 1993) y el N total en leche se determinó a partir de la fórmula:

$\mathrm{N}$ total de leche $\left(\mathrm{g} / \mathrm{L}^{-1}\right)=($ Proteína de leche / 6,38) $+\mathrm{N}$ ureico en leche

Fecas. Para la determinación de la cantidad de fecas excretadas se utilizó $\mathrm{Cr}_{2} \mathrm{O}_{3}$ como marcador. Se suministraron a cada vaca $3 \mathrm{~g}$ diarios de este marcador, en 2 dosis de 1,5 g, dentro de cápsulas de almidón que eran suministradas intrarruminalmente con un aplicador, vía oral. El $\mathrm{Cr}_{2} \mathrm{O}_{3}$ se administró durante 11 días, de los cuales los primeros 6 se consideraban de saturación del tracto gastrointestinal, mientras que durante los últimos 5 días se tomaban las muestras de fecas para las deter-minaciones. Luego de cada ordeña, aproximadamente $200 \mathrm{~g}$ de fecas se extrajeron desde el recto. Estas eran congeladas para luego ser analizadas en MS, N y óxido crómico. La MS se determinó por liofilización y el $\mathrm{N}$ por Kjeldahl, mientras que el óxido crómico se cuantificó por espectrofotometría de absorción atómica (Williams y col 1962). La producción de MS fecal se determinó mediante la fórmula:

\section{MS fecal = 1000/concentración de óxido crómico}

Orina. En cada uno de los dos períodos se realizaron colectas totales de orina cada 90 minutos, durante dos días, para determinar el volumen diario excretado. La colecta se realizó de la micción natural inducida mediante estimulación vulvar. Un $3 \%$ del volumen de orina obtenido se guardó preservado con ácido sulfúrico al 10\%, con la cual se formó una submuestra representativa del día a fin de determinar MS por liofilización, $\mathrm{N}$-urinario mediante Kjeldahl y urea mediante la técnica colorimétrica de ureasa-Berthelot. El volumen y concentración de $\mathrm{N}$ promedio de estos dos días fue utilizado para el resto del período en cada uno de los ensayos.

Balance de N. Tomando en cuenta todas las variables anteriores, se calculó el balance de $\mathrm{N}$ de acuerdo a la fórmula:

Balance de $\mathrm{N}=\mathrm{N}$ consumido $-\mathrm{N}$ secretado en leche $-\mathrm{N}$ excretado en fecas $-\mathrm{N}$ excretado en orina
Ganancia de peso. Las vacas fueron pesadas al inicio y al final del ensayo, y posterior a la ordeña de la mañana. La ganancia de peso se determinó de acuerdo a la diferencia de peso en el período. El N depositado en ganancia de peso se estimó de acuerdo a la fórmula:

$$
\begin{gathered}
\mathrm{N} \text { depositado }(\mathrm{g} / \mathrm{d})=\text { variación en peso vivo }(\mathrm{kg}) \\
\mathrm{x} 26(\mathrm{NRC} 1989)
\end{gathered}
$$

\section{ANALISIS ESTADISTICO}

Los datos se analizaron con el paquete estadístico SAS (SAS 1995), mediante el procedimiento GLM, de acuerdo al siguiente modelo:

$$
Y_{i j k}=\mu+T_{i}+P_{j}+B_{k}+E_{i j k}
$$

Siendo:

$\mathrm{Y}=$ variable dependiente

$\mu=$ media de $\mathrm{Y}$, considerada diariamente

$\mathrm{T}_{\mathrm{i}}=$ efecto del tratamiento $(\mathrm{i}=1,2,3,4)$

$\mathrm{P}_{\mathrm{j}}=$ efecto del período $(\mathrm{j}=1,2)$

$\mathrm{B}_{\mathrm{k}}=$ efecto del bloque $(\mathrm{k}=1,2)$

$\mathrm{E}_{\mathrm{ijk}}=$ error residual

Las variables, cuyo análisis de varianza dio como resultado diferencias significativas $(\mathrm{P}<0,05)$, fueron sometidas a una prueba de comparación de medias, utilizándose la prueba de contrastes ortogonales (95\%). Los resultados son presentados como medias aritméticas con el error estándar correspondiente

\section{RESULTADOS}

Consumo, producción y composición de leche. Las vacas que consumieron dietas con mayor contenido de PNDR tuvieron un menor consumo de alimentos y de $\mathrm{N}$ $(\mathrm{P}<0,05)$ que las vacas alimentadas con menos PNDR. La producción de leche total y de proteína, y su composición en cuanto a porcentaje de proteína y contenido de $\mathrm{N}$ ureico en leche, fue similar para los cuatro grupos de animales $(\mathrm{P}>0,05)$ (cuadro 2).

Excreción de purines y composición de orina. La excreción diaria de orina y de fecas fue mayor en las dietas A1 y A2 ( $\mathrm{P}<0,01$ y $\mathrm{P}<0,05$ respectivamente), existiendo una interacción entre cantidad de ensilaje de alfalfa $\mathrm{x}$ harina de pescado $(\mathrm{P}<0,05)$ para la excreción de orina, mientras que el contenido de MS de la orina fue menor $(\mathrm{P}<0,05)$ en las dietas no suplementadas con harina de pescado (dietas A) (cuadro 3).

La concentración de urea en la orina fue mayor $(\mathrm{P}<0,01)$ en los animales que recibieron harina de pescado, si bien la cantidad diaria de $\mathrm{N}$ excretada como urea fue mayor en las vacas con las dietas A. El porcentaje del $\mathrm{N}$ urinario proveniente de la urea fue similar entre las 
Cuadro 2. Efecto del incremento de PNDR en la dieta mediante harina de pescado (dietas B) sobre el consumo y la producción y composición de leche en vacas lecheras alimentadas con dietas basadas en ensilajes de pradera en 45 y $38 \%$ y de alfalfa en 20 y $27 \%$ (dietas 1 y 2 respectivamente).

Effect of increasing RUP on intake, milk production and composition of milk in dairy cows fed high silage diets.

\begin{tabular}{|c|c|c|c|c|c|c|c|c|c|}
\hline \multirow[t]{2}{*}{ Dieta } & \multirow[t]{2}{*}{$\mathrm{A} 1$} & B1 & $\mathrm{A} 2$ & \multirow[t]{2}{*}{ B2 } & \multicolumn{5}{|c|}{ Contrastes } \\
\hline & & \multicolumn{2}{|c|}{ Promedios } & & e.e. & 1 & 2 & 3 & $\begin{array}{c}\mathrm{I} \\
\mathrm{A}^{* \mathrm{P}}\end{array}$ \\
\hline \multicolumn{10}{|l|}{ Consumo } \\
\hline $\mathrm{MS}^{\mathrm{v}}$ & 21,5 & 20,3 & 21,5 & 19,6 & 0,46 & NS & $*$ & $* *$ & NS \\
\hline $\mathrm{PNDR}^{\mathrm{w}}$ & 798 & 910 & 808 & 947 & 26 & $* *$ & $* *$ & $* *$ & NS \\
\hline $\mathrm{N}^{\mathrm{w}}$ & 549 & 514 & 554 & 509 & 14 & NS & NS & $*$ & NS \\
\hline \multicolumn{10}{|l|}{ Leche } \\
\hline Producción ${ }^{\mathrm{x}}$ & 25,3 & 26,6 & 25,8 & 26,3 & 0,54 & NS & NS & NS & NS \\
\hline Proteína ${ }^{\mathrm{y}}$ & 3,16 & 3,18 & 3,08 & 3,13 & 0,03 & NS & NS & NS & NS \\
\hline Prod. Prot ${ }^{w}$ & 799 & 843 & 797 & 822 & 18,6 & NS & NS & NS & NS \\
\hline $\mathrm{N}$ ureico ${ }^{\mathrm{z}}$ & 14,9 & 13,9 & 15,4 & 14,1 & 0,34 & NS & NS & NS & NS \\
\hline
\end{tabular}

v: $\mathrm{kg} \mathrm{d}^{-1} ; \mathrm{w}: \mathrm{g} \mathrm{d}^{-1} ; \mathrm{x}: \mathrm{L} \mathrm{d}^{-1} ; \mathrm{y}: \% ; \mathrm{z}: \mathrm{mg} \mathrm{dL}^{-1}$.

Contrastes: 1: A1 vs. B1; 2: A2 vs. B2; 3; A vs. B; I A*P: interacción nivel de ensilaje de alfalfa y de PNDR.

$*$ : $\mathrm{P}<0,05 ; * *$ : $\mathrm{P}<0,01 ; \mathrm{NS}: \mathrm{P}>0,05$.

Cuadro 3. Efecto del incremento de PNDR en la dieta mediante harina de pescado sobre la excreción y composición de las fecas y orina de vacas lecheras alimentadas con dietas basadas en ensilajes de pradera en 45 y $38 \%$ y de alfalfa en 20 y $27 \%$ (dietas 1 y 2 respectivamente).

Effect of increasing RUP on excretion and composition of faeces and urine in dairy cows fed high silage diets.

\begin{tabular}{|c|c|c|c|c|c|c|c|c|c|}
\hline \multirow[t]{2}{*}{ Dieta } & \multirow[t]{2}{*}{$\mathrm{A} 1$} & B1 & $\mathrm{A} 2$ & B2 & \multicolumn{5}{|c|}{ Contrastes } \\
\hline & & \multicolumn{2}{|c|}{ Promedios } & & e.e & 1 & 2 & 3 & $\begin{array}{c}\mathrm{I} \\
\mathrm{A}^{*} \mathrm{P} \\
\end{array}$ \\
\hline \multicolumn{10}{|l|}{ Fecas } \\
\hline Peso $^{v}$ & 7,64 & 7,31 & 7,54 & 6,76 & 0,19 & NS & $*$ & $*$ & NS \\
\hline $\mathrm{MS}^{\mathrm{w}}$ & 64,4 & 64,0 & 64,8 & 65,4 & 0,71 & NS & NS & NS & NS \\
\hline \multicolumn{10}{|l|}{ Orina } \\
\hline Volumen ${ }^{\mathrm{X}}$ & 23,1 & 21,4 & 23,1 & 19,3 & 0,51 & NS & $* *$ & $* *$ & $*$ \\
\hline $\mathrm{MS}^{\mathrm{w}}$ & 5,40 & 5,65 & 5,45 & 5,65 & 0,09 & NS & NS & $*$ & NS \\
\hline Urea $^{y}$ & 18,1 & 18,7 & 18,3 & 19,9 & 0,38 & NS & $* *$ & $* *$ & NS \\
\hline N-ureico ${ }^{z}$ & 193 & 185 & 196 & 178 & 4,43 & NS & NS & NS & NS \\
\hline $\begin{array}{l}\mathrm{N} \text { urinario/ } \mathrm{N} \\
\text { ureico }\end{array}$ & 83,2 & 81,8 & 80,4 & 84,8 & 0,82 & NS & $* *$ & NS & $* *$ \\
\hline
\end{tabular}

v: $\mathrm{kg} \mathrm{d}^{-1} ; \mathrm{w}: \% ; \mathrm{x}: \mathrm{L} \mathrm{d}^{-1} ; \mathrm{y}: \mathrm{g} \mathrm{L}^{-1} ; \mathrm{z}: \mathrm{g} \mathrm{d}^{-1}$.

Contrastes: 1: A1 vs. B1; 2: A2 vs. B2; 3: A vs. B; I A*P: interacción nivel de ensilaje de alfalfa y de PNDR.

$*$ : $\mathrm{P}<0,05 ; * *: \mathrm{P}<0,01 ; \mathrm{NS}: \mathrm{P}>0,05$.

dietas A y B, constituyendo más del $80 \%$ del $\mathrm{N}$ excretado por esa vía (cuadro 3).

Eficiencia de utilización del N consumido. La digestibilidad aparente de las raciones fue similar en los cuatro grupos, al igual que la excreción de $\mathrm{N}$ en leche
$(\mathrm{P}>0,05)$. Sin embargo, las dietas con mayores contenidos de PNDR (dietas $\mathrm{B}$ ) resultaron en una menor excreción de $\mathrm{N}$ vía orina $(\mathrm{P}<0,01)$ y de $\mathrm{N}$ total en purines (fecas más orina) $(\mathrm{P}<0,05)$ y tuvieron un menor balance de $\mathrm{N}(\mathrm{P}<0,05)$. Existió una interacción entre el contenido de ensilaje de alfalfa y la suplementación con harina de pescado para el $\mathrm{N}$ excretado en orina $(\mathrm{P}<0,05)$. Los grupos con mayor aporte de PNDR (dietas B) utilizaron una cantidad mayor de $\mathrm{N}$ en funciones productivas (leche más ganancia de peso, expresado como g día ${ }^{-1}$ ) ( $\mathrm{P}<0,01$; cuadro 4$)$, existiendo una interacción entre el ensilaje de alfalfa y la suplementación con PNDR $(\mathrm{P}<0,01)$.

No existieron diferencias en la relación entre el $\mathrm{N}$ excretado por fecas y orina y el $\mathrm{N}$ consumido para los tratamientos $(\mathrm{P}>0,05)$. Sin embargo, la suplementación con harina de pescado como fuente de PNDR incrementó la cantidad de $\mathrm{N}$ secretado en leche y el destinado a funciones productivas (leche más ganancia de peso) $(\mathrm{P}<0,01)$. Al analizar la relación entre la cantidad de $\mathrm{N}$ excretado en fecas y orina por litro de leche producido, se excretó en purines menos $\mathrm{N}$ en las dietas $\mathrm{B}(\mathrm{P}<0,01)$, es decir, en las suplementadas con harina de pescado. La relación entre $\mathrm{N}$ destinado a funciones productivas (leche más ganancia de peso) y $\mathrm{N}$ excretado en fecas y orina es un indicador objetivo de esta eficiencia que resume el objetivo de este ensayo. A pesar de la baja eficiencia de utilización, en las dietas $\mathrm{B}$ este $\mathrm{N}$ destinado a funciones productivas fue mayor $(\mathrm{P}<0,01)$.

\section{DISCUSION}

Consumo, producción y composición de leche y ganancia de peso. El menor consumo que se produjo con las dietas B podría estar relacionado con la suplementación con harina de pescado que contenía un elevado porcentaje de aceite (EE: 10,75\%), coincidente con lo descrito en otros trabajos (Hussein y Jordan 1991). A pesar de este menor consumo de MS y de N, no existieron diferencias en la producción de leche y de proteína, es decir, que los animales de los grupos A, con menores aportes de PNDR, generaron igual producción y composición de leche con un consumo mayor de MS y de N. Esto hace pensar que en las dietas A las limitantes estuvieran en el aporte de PNDR. Eso concuerda con lo descrito con Dhiman y Satter (1993), quienes observaron que en dietas basadas en ensilaje de alfalfa el nutriente que limitó la producción fue la proteína y, en particular, la fracción PNDR. El contenido de $\mathrm{N}$ ureico en leche no fue diferente, y estuvo por debajo de los valores considerados críticos $\left(19 \mathrm{mg} \mathrm{dl}^{-1}\right.$ ) por autores americanos (Baker y col 1995). Se describe que el contenido de PNDR de la dieta influye en la concentración del N-ureico de la leche (Baker y col 1995), situación que no fue apreciada en esta experiencia, lo que podría estar asociado a que la mayor variación se expresó en el contenido de PNDR de 
Cuadro 4. Efecto del incremento de PNDR en la dieta mediante harina de pescado sobre el balance y eficiencia de la utilización del $\mathrm{N}$ en vacas lecheras alimentadas con dietas basadas en ensilaje de pradera en 45 y $38 \%$ y de alfalfa en 20 y $27 \%$ (dietas 1 y 2 respectivamente).

Effect of increasing RUP on nitrogen balance and efficiency of the use of $\mathrm{N}$ in dairy cows fed high silage diets.

\begin{tabular}{|c|c|c|c|c|c|c|c|c|c|}
\hline \multirow[t]{2}{*}{ Dieta } & $\mathrm{A} 1$ & B1 & $\mathrm{A} 2$ & B2 & \multirow[b]{2}{*}{ e.e } & \multicolumn{3}{|c|}{ Contrastes } & \multirow{2}{*}{$3 \stackrel{\mathrm{I}}{\mathrm{A}} \mathrm{A} \mathrm{P}$} \\
\hline & \multicolumn{4}{|c|}{ Promedios } & & 1 & 2 & 3 & \\
\hline \multicolumn{10}{|l|}{$\mathrm{N}$ excretado (g/d) } \\
\hline Fecas & 171 & 164 & 169 & 159 & 3,8 & NS & NS & NS & NS \\
\hline $\begin{array}{l}\text { Dig. } \\
\text { aparente N }\end{array}$ & 69 & 68 & 70 & 68 & 0,7 & NS & NS & NS & NS \\
\hline Orina & 231 & 227 & 234 & 210 & 5,3 & NS & $* *$ & $* *$ & * \\
\hline $\begin{array}{l}\text { Fecas más } \\
\text { orina }\end{array}$ & 402 & 391 & 403 & 369 & 8,0 & NS & $* *$ & $*$ & NS \\
\hline Leche & 129 & 136 & 129 & 133 & 3,0 & NS & NS & NS & NS \\
\hline Balance de N & 18,4 & $-13,5$ & 22,5 & 7,6 & 9,1 & $*$ & NS & $*$ & NS \\
\hline $\begin{array}{l}\text { Ganancia } \\
\text { de peso }\end{array}$ & 6,05 & 33,2 & 11,6 & 12,5 & 3,5 & $* *$ & NS & $* *$ & $* *$ \\
\hline N productivo $\otimes$ & 135 & 169 & 140 & 145 & 4,9 & $* *$ & NS & $* *$ & $* *$ \\
\hline \multicolumn{10}{|l|}{$\begin{array}{l}\text { N excretado } \\
\text { (g/kg de } \mathrm{N} \\
\text { consumido) }\end{array}$} \\
\hline Fecas & 313 & 323 & 305 & 316 & 7,0 & NS & NS & NS & NS \\
\hline Orina & 422 & 444 & 424 & 420 & 9,9 & NS & NS & NS & NS \\
\hline Leche & 229 & 261 & 225 & 256 & 5,8 & $* *$ & $* *$ & $* *$ & NS \\
\hline N productivo $\otimes$ & 247 & 338 & 252 & 287 & 18,2 & $* *$ & $* *$ & $* *$ & $* *$ \\
\hline \multicolumn{10}{|l|}{ Relación } \\
\hline $\begin{array}{l}\text { N excretado total/ } \\
\text { L leche \# }\end{array}$ & 15,9 & 14,8 & 15,7 & 14,3 & 0,4 & $*$ & * & $* *$ & NS \\
\hline $\begin{array}{l}\text { N Productivo /N } \\
\text { excretado \#\# }\end{array}$ & 0,34 & 0,44 & 0,35 & 0,40 & 0,02 & $* *$ & $* *$ & $* *$ & $* *$ \\
\hline
\end{tabular}

Contrastes: 1: A1 vs. B1; 2: A2 vs. B2; 3; A vs. B; I A*P: interacción nivel de ensilaje de alfalfa y de PNDR.

$\otimes: \mathrm{N}$ secretado en leche más $\mathrm{N}$ depositado en ganancia de peso.

\#: relación entre $\mathrm{N}$ excretado en purines y producción de leche $\left(\mathrm{g} \mathrm{Lt}^{-1}\right)$; \#\#: relación entre $\mathrm{N}$ productivo ( $\mathrm{N}$ en leche más ganancia diaria) y $\mathrm{N}$ excretado ( $\mathrm{N}$ fecal más urinario $\mathrm{N}$ ) $\left(\mathrm{g} \mathrm{g}^{-1}\right)$.

*: $\mathrm{P}<0,05 ; * *: \mathrm{P}<0,01 ; \mathrm{NS}: \mathrm{P}>0,05$.

la dieta, situación similar a la descrita por otros autores (Gonda y Lindberg 1994).

Excreción de purines y composición de orina. La producción de orina se explica, en parte, por la excreción de urea, con la cual estuvo fuertemente relacionada la excreción de orina $(\mathrm{r}=0,79 ; \mathrm{P}<0,01)$, lo que concuerda con Gonda y Lindberg (1994) y con Bannink y col (1999). Estos últimos autores, en una revisión de 10 ensayos, con una amplia variación de dietas y niveles de producción, también consideraron la excreción de minerales como $\mathrm{Na}$ y K, y observaron que cerca del $90 \%$ del volumen de orina producida se explica a través de las cantidades de $\mathrm{N}$, Na y K excretados en la orina. En nuestro trabajo, la suplementación con harina de pescado aumentó la concentración de urea en orina, lo que discrepa con lo descrito por Gonda y Lindberg (1994), quienes afirman que la degradabilidad de la proteína de la dieta no afecta la concentración de urea en orina. La mayor concentración de urea en la orina de los grupos suplementados con PNDR se compensa con el menor volumen de orina excretado, lo que conduce a que la cantidad de $\mathrm{N}$ ureico de la orina sea similar.

La excreción de fecas fue afectada por la suplementación con una fuente de PNDR, aunque es probable que dicho efecto se relacione principalmente al menor consumo de MS que tuvieron los animales que se suplementaron con PNDR. Sobre la base de nuestros resultados, parece claro que la suplementación con una fuente de PNDR, que permitió un uso más eficiente de los nutrientes, estuvo asociada a una menor excreción de fecas y de orina.

Eficiencia de utilización del $N$ consumido. Los resultados de excreción fecal y urinaria de $\mathrm{N}$ reflejan las diferencias en consumo de $\mathrm{MS}$ y de $\mathrm{N}$ total y concuerdan con otros resultados (Wohlt y col 1991; Maltz y Silanikove 1996). En nuestro trabajo, la excreción de $\mathrm{N}$ urinario estuvo relacionada al consumo de $\mathrm{N}$ y al $\mathrm{N}$ ureico en leche a partir de una ecuación de regresión, teniendo en cuenta estas variables, las que se detallan a continuación:

$$
\begin{aligned}
& \mathrm{N} \text { urinario }=123,3-1,45^{*} \mathrm{~N} \text { ureico en leche } \\
& +0,23 * \mathrm{~N} \text { consumido }\left(\mathrm{r}^{2}=0,34 ; \mathrm{P}<0,01\right)
\end{aligned}
$$

Esta relación es similar a la descrita por Jonker y col (1999). Jonker y col (2002) obtuvieron valores de $\mathrm{N}$ urinario menores que los nuestros en 454 lecherías de EE.UU. La menor excreción de $\mathrm{N}$ urinario observada en las dietas B se atribuye al menor consumo de $\mathrm{N}$ con estas dietas, ya que al expresar la excreción de $\mathrm{N}$ en orina como $\mathrm{g} / \mathrm{kg}$ de $\mathrm{N}$ consumido estas diferencias desaparecen. La interacción que existe entre el nivel de ensilaje de alfalfa por harina de pescado discrepa con lo reportado por Broderick (2004), quien señala que los ensilajes de alfalfa tienen altos niveles de NNP, por lo que con ellos se incrementa la excreción de $\mathrm{N}$ urinario. El $\mathrm{N}$ destinado a funciones productivas fue mayor en las dietas suplementadas con harina de pescado. Este efecto reafirma la posibilidad de una restricción de aminoácidos a nivel intestinal al alimentar con dietas basadas en ensilajes (Santos y col 1998) y, en particular, en dietas basadas en ensilaje de alfalfa (Dhiman y Satter 1993). La utilización del N consumido es particularmente importante, porque la proteína y, por lo tanto, el $\mathrm{N}$ de la dieta es uno de los nutrientes más caros y porque, además, su excreción es una importante fuente de polución y de contaminación de aguas subterráneas con nitratos y de la atmósfera con amoníaco (Tamminga 1996). 
Existieron diferencias en el $\mathrm{N}$ utilizado en funciones productivas (secretado en leche más ganancia de peso). Es interesante notar que, independientemente del tratamiento, la eficiencia de utilización del $\mathrm{N}$ consumido para producción de leche fue baja y fluctuó entre 230 y $260 \mathrm{~g} / \mathrm{kg}$ del $\mathrm{N}$ consumido. Estos valores son, sin embargo, más altos que los reportados por Cushnahan y col (1995), pero similares a los de otros autores (Van Vuuren y col 1993; Dewhurst y col 1996; Castillo y col 2001) para $\mathrm{N}$ secretado en leche y menores que los reportados por Frank y Swensson (2002). Asimismo, concuerdan con los de Robinson y col (1995), quienes al ajustar el balance aminoacídico con una suplementación con metionina y lisina protegidas obtuvieron, al igual que en este trabajo, una mayor proporción del $\mathrm{N}$ consumido que se excreta en leche. Estas diferencias en la eficiencia de utilización del $\mathrm{N}$ para proteína láctea se explica por los mayores niveles de PNDR así como por el incremento en el aporte de metionina y lisina (Santos y col 1998). En nuestro trabajo, el incremento en los aportes de PNDR por la inclusión de la harina de pescado, incrementó tanto la utilización del N consumido para producción de proteína láctea como para funciones productivas, lo que hace pensar que la limitante de estas dietas estaría en el aporte de proteína metabolizable, según lo descrito por Broderick (2004).

No existieron diferencias entre tratamientos en la proporción del $\mathrm{N}$ consumido que fue excretado como $\mathrm{N}$ fecal y $\mathrm{N}$ urinario (expresado como $\mathrm{g} / \mathrm{kg}$ ), con valores similares a los observados en otros trabajos (Van Vuuren y col 1993). Estos valores son menores que los descritos en una experiencia con animales alimentados únicamente con ensilajes, probablemente porque la producción de $\mathrm{NH}_{3}$ ruminal debe haber sido mayor (Cushnahan y col (1995). De igual forma, con dietas basadas en heno se ha descrito una menor excreción del $\mathrm{N}$ consumido, expresada en \% (61,4 a 65,9 y 58 a $67 \%$ respectivamente) y una mayor relación entre $\mathrm{N}$ productivo y $\mathrm{N}$ excretado (0,51 a 0,64 y 0,44 a 0,59, respectivamente) (Susmel y col 1995; Lines y Weiss 1996), lo que se debería a que los animales eran alimentados con heno de festuca y de alfalfa respectivamente, forrajes que poseen una menor tasa de degradación ruminal del $\mathrm{N}$ que los ensilajes. Al analizar la relación $\mathrm{N}$ excretado por litro de leche producida, se observó un efecto positivo de la inclusión de harina de pescado, ya que las dietas que la contenían (dietas B) generaron una menor excreción de $\mathrm{N}$ por litro de leche producida. Además, la relación entre el N utilizado en funciones productivas (leche más ganancia de peso) y el $\mathrm{N}$ excretado en fecas y orina fue un buen indicador de esta mejor eficiencia. Cuando consideramos este parámetro, las ventajas de suplementar dietas basadas en ensilajes con harina de pescado fueron evidentes, ya que en todos los casos estas dietas produjeron significativamente más $\mathrm{N}$ recuperado en funciones productivas por $\mathrm{kg}$ de $\mathrm{N}$ excretado.
En resumen, los resultados indican que la corrección con harina de pescado, de un déficit de PNDR presente en dietas ricas en ensilajes (de pradera y alfalfa), permitió una mayor eficiencia de utilización del $\mathrm{N}$ consumido en funciones de producción y se disminuyó la contaminación potencial a través de una reducción de la excreción de $\mathrm{N}$.

\section{RESUMEN}

El objetivo de este trabajo fue establecer los efectos de diferentes niveles de PNDR en dietas con alto contenido de ensilajes sobre la utilización y excreción del $\mathrm{N}$ consumido. El ensayo tuvo 2 períodos de 14 días, utilizándose 8 vacas en lactancia en un diseño de cuadrados latinos incompletos. Las dietas identificadas como A1 y B1 tenían: base materia seca (MS), un $45 \%$ de ensilaje de pradera permanente (PP), un $20 \%$ de ensilaje de alfalfa (EA) y un $35 \%$ de concentrado, y las A2 y B2 un 38\% de ensilaje de PP, un $27 \%$ de EA y un $35 \%$ de concentrado. Las dietas B se ajustaron a las recomendaciones de PNDR de NRC (1989), mientras que en las dietas A se permitió un déficit de esta fracción de hasta un 20\%. Las dietas B (suplementadas con harina de pescado) generaron un menor consumo de $\mathrm{N}$ sin afectar la producción de leche ni su composición. Sin embargo, las dietas adecuadas en PNDR redujeron la excreción de fecas y de orina así como de la cantidad de urea excretada en orina. Al evaluar la excreción de $\mathrm{N}$, los grupos suplementados con PNDR excretaron menos $\mathrm{N}$ vía fecas y orina y utilizaron más $\mathrm{N}$ en funciones de producción (leche más ganancia de peso). Al evaluar la eficiencia de utilización del $\mathrm{N}$ consumido (expresado como g/kg de $\mathrm{N}$ consumido), los grupos suplementados con PNDR excretaron más $\mathrm{N}$ consumido en la leche y en ganancia de peso. Asimismo, el $\mathrm{N}$ excretado, expresado como $\mathrm{N}$ por litro de leche producida, fue menor en las dietas suplementadas con harina de pescado. Se concluye que al corregir un déficit de PNDR en dietas ricas en ensilaje con una adecuada suplementación con harina de pescado, ajustada a los requerimientos animales, se permite una buena utilización del $\mathrm{N}$ consumido, reduciendo las excreciones de $\mathrm{N}$ al medio ambiente.

\section{AGRADECIMIENTOS}

A los profesores R. Smith, F. Mujica y J. Piaggio por su asesoramiento en el diseño del experimento y análisis de los resultados, así como a los estudiantes A. Cox, P. Alarcón y J. Céspedes por su colaboración en la alimentación de los animales y toma de datos. Al personal del Predio Experimental "Vista Alegre" por el cuidado y manejo de los animales.

\section{REFERENCIAS}

Anrique R, X Valderrama, R Fuchslocher. 1995. Tabla de composición de alimentos para el ganado de la zona sur. Ed. Fundación Fondo de Investigación Agropecuario. Ministerio de Agricultura. Association of Official Analytical Chemists. 1996. Official methods of analysis. $16^{\text {th }}$ ed. AOAC, Arlington, VA.

Baker L D, J D Fergusson, W Chalupa. 1995. Responses in urea and true protein of milk to different protein feeding schemes for dairy cows. J Dairy Sci 78, 2424-2434.

Bannink A, H Valk, A M Van Vuuren. 1999. Intake and excretion of sodium, potassium, and nitrogen and the effects on urine production by lactating dairy cows. J Dairy Sci 82, 1008-1018.

Broderick G. 2004. Effect of low level monensin supplementation on the production of dairy cows fed alfalfa silage. J Dairy Sci 87, 359-368.

Butler W R. 2000. Nutritional interactions with reproductive performance in dairy cattle. Anim Reprod Sci 60-61, 449-457. 
Castillo A R. 1999. Improving nitrogen utilization in dairy cows. Ph.D. Thesis. University of Reading, UK.

Castillo A R, E Kebreath, D E Beever, J H Barbi, J D Sutton, H C Kirby, J. France. 2001. The effect of protein supplementation on nitrogen utilization in lactating dairy cows fed grass silage diets. J Anim Sci 79, 247-253.

Cushnahan A, C S Mayne, E F Unsworth. 1995. Effects of ensilage on performance and nutrient utilization by dairy cattle. 2 . Nutrient metabolism and rumen fermentation. Anim Sci 60, 347-359.

Dewhurst R J, A M Mitton, N W Ofer, C Thomas. 1996. Effects of the composition of grass silages on milk production and nitrogen utilization by dairy cows. Anim Sci 62, 25-34.

Dhiman T R, L D Satter. 1993. Protein as the first limiting nutrient for lactating dairy cows fed high proportions of good quality alfalfa silage. J Dairy Sci 76, 1960-1968.

Dou Z, R Kohn, J Fergusson, R Boston, J Newbold. 1996. Managing nitrogen on dairy farms: an integrated approach 1 . Model description. J Dairy Sci 79, 2071-2080.

Frank B, C Swensson. 2002. Relationship between content of crude protein in rations for dairy cows and milk yield, concentrations of urea in milk and ammonia emissions. J Dairy Sci 85, 1829-1838.

Gill J. 1981. Experimental design and analysis. Cornell University Press. Cornell, Pp 473.

Gonda H, J Linndberg. 1994. Evaluation of dietary nitrogen utilization in dairy cows based on urea concentrations in blood, urine and milk and on urinary concentrations of purine derivatives. Acta Agric Scand, Sect A, Anim Prod 44, 236-245.

Grummer R, K Slark, S Bertics, M Luck, J Barmore. 1996. Soybeans versus animal sources of rumen-undegradable protein and fat for early lactation dairy cows. J Dairy Sci 79, 1809-1816.

Hussein H S, R M Jordan. 1991. Fish meal as a protein supplement in ruminants diets: a review. J Anim Sci 69, 2147-2156.

Jonker J, R Kohn, R Erdman. 1999. Milk urea nitrogen target concentrations for lactating dairy cows fed according to National Research Council recommendations. J Dairy Sci 82, 1261-1273.

Jonker J, R Koh, J High. 2002. Dairy herd management practices that impact nitrogen utilization efficiency. J Dairy Sci 85,1218-1226.

Lines L W, W Weiss. 1996. Use of nitrogen from ammoniated alfalfa hay, urea, soybean meal, and animal protein meal by lactating dairy cows. J Dairy Sci 79, 1992-1999.

Maltz E, N Silanikove. 1996. Kidney function and nitrogen balance of high yielding dairy cows at the onset of lactation. J Dairy Sci 79, 1621-1626.

National Research Council. 1989. Nutrients requirements for dairy cattle. $6^{\text {th }}$ rev. ed. National Academy Press. Washington, DC.

Nousiainen J, K Shingfield, P Huthtanen. 2004. Evaluation of milk urea nitrogen as a diagnostic of protein feeding. J Dairy Sci 87, 386-398.

O’Mara F P, J Murphy, M Rath. 1998. Effect of amount of dietary supplement and source of protein on milk production, ruminal fermentation, and nutrient flows in dairy cows. J Dairy Sci 81, 2430-2439.

Pell A. 1992. Does rations balancing affect nutrient mana-gement? Proc Cornell Nut Conf Feed Manuf, Pp 23-31.
Pitt R. 1995. Nutrient flows on dairy farms: a case study. Proc Cornell Nut Conf Feed Manuf, Pp 153-160.

Polan C, G Cozzi, P Berzaghi, I Andrighetto. 1997. A blend of animal and cereal protein or fish meal as partial replacement for soybean meal in the diets of lactating Holstein cows. J Dairy Sci 80, 160-166.

Robinson P H, A Fredeen, W Chalupa, W Julien, H Sato, T Fujieda, H Suzuki. 1995. Ruminally protected lysine and methionine for lactating diary cows fed a diet designed to met requirements for microbial and postruminal protein. J Dairy Sci 78, 582-594.

Roseler D, J Fergusson, C Sniffen, J Herrema. 1993. Dietary protein degradability effects on plasma and milk urea nitrogen and milk nonprotein nitrogen in Holstein cows. J Dairy Sci 76, 525-534.

Santos F A, J E Santos, C Theurer, J T Huber. 1998. Effects of rumenundegradable protein on dairy cow performance: a 12 year literature review. J Dairy Sci 81, 3182-3213.

SAS USER'S GUIDE: Statistics. Version 1995. SAS Inst. Inc., Cary, NC.

Schepers A, R Meijer. 1998. Evaluation of the utilization of dietary nitrogen by dairy cows based on urea concentration in milk. J Dairy Sci 81, 579-584.

Spedding C R W. 1995. Sustainability in animal production systems. Anim Sci 61, 1-8.

Susmel P, M Spanghero, B Stefanon, C Mills. 1995. Nitrogen balance and partitioning of some nitrogen catabolites in milk and urine of lactating cows. Livest Prod Sci 44, 207-219.

Tamminga S. 1992. Nutrition management of dairy cows as a contribution to pollution control. J Dairy Sci 75, 345-57.

Tamminga S. 1996. A review on environmental impacts of nutritional strategies in ruminants. J Anim Sci 74, 3112-3124.

Tilley J, R Terry. 1963. A two stage technique for the in vitro digestion of forage crops. J British Grassland Soc 18, 104-111.

Van Soest P J, J Robertson, B Lewis. 1991. Methods for dietary fiber, neutral detergent fiber, and nonstarch polysaccharides in relation to animal nutrition. J Dairy Sci 74, 3583-3595.

Van Straalen W. 1995. Modelling of nitrogen flow and excretion in dairy cows. PhD. Thesis. Wageningen Agricultural University. The Netherlands.

Van Vuuren A M, C J Van Der Koelen, H Valk, H De Visser. 1993. Effects of partial replacement of ryegrass by low protein feeds on rumen fermentation and nitrogen loss by dairy cows. J Dairy Sci 76, 2982-2993.

Williams C H, D David, O Lismaa. 1962. The determination of chromic oxide in faeces samples by atomic absorption spectrophotometry. J Agric Sci Camb 59, 381-385

Wittwer F G, H Opitz, J Reyes, P C Contreras, H Böhmwald. 1993. Determinación de urea en muestras de leche de rebaños bovinos para el diagnóstico de desbalance nutricional. Arch Med Vet 25, $165-172$

Wohlt J E, S Chmiel, P Zajac, L Baker, D Blethen, J Evans. 1991. Dry matter intake, milk yield and composition, and nitrogen use in Holstein cows fed soybean, fish, or corn gluten meals. J Dairy Sci 74, 1609-1622. 